\title{
Tangible Interaction with Light: A Review
}

\author{
Yanhong Li *D, Beat Rossmy and Heinrich Hußmann \\ Media Informatics, Ludwig-Maximilians-Universität München, 80337 München, Germany; \\ beat.rossmy@ifi.lmu.de (B.R.); hussmann@ifi.lmu.de (H.H.) \\ * Correspondence: yanhong.li@ifi.lmu.de
}

Received: 30 June 2020; Accepted: 25 September 2020; Published: 5 October 2020

check for updates

\begin{abstract}
Light is an important means of information representation and feedback in Human-Computer Interaction (HCI), and light-emitting interaction elements are omni-present. We address here the interplay of light and tangible interaction with specifically designed objects. The goal of such designs is to support an embodied, emotional and engaged interaction experience integrated into the physical surroundings. The specific combination of tangible interaction and light as a medium is used in several approaches, but a systematic overview of this research area still does not exist. In order to understand the essence, process and result of tangible interaction with light, we conducted a systematic literature review of 169 studies of tangible interaction with light over the past 20 years. Our results provide a demographic overview of the research, but foremost analyze their concepts, purposes, conceptual frameworks, user contexts, interaction behaviors and problems addressed by tangible light. Three important findings were obtained: (1) Tangible interaction with light has been used for diverse purposes, contexts and interactions; (2) Tangible light has addressed problems: weak interaction, don't know how to interact, interaction lacks innovation, collaborative interaction, remote tangible interaction, and emotional interaction; (3) Current research in this area can be classified as "wild theory" in conceptual research frameworks, which means it emphasizes very much on innovation. The most important contribution of this work is the systematic review in itself, but the findings of our work also give some indications on new ways and future trends for tangible interaction, when combined with light as a medium.
\end{abstract}

Keywords: HCI; tangible interaction; user interface; tangible user interface; TUI; tangible light; human-light interaction

\section{Introduction}

"God saw that the light was good, and he separated the light from the darkness." Light is essential to humans not only for lighting and decoration, but also for information communication, which refers to visible light communication [1]. Since more than $80 \%$ of our perception, learning, cognition, and activities are mediated through vision, light is an important medium for Human-Computer Interaction (HCI). In this work, we focus on a very specific topic, which is the combination of tangible interaction using physical objects with light as a medium to output information or to allow user input via light. This means to integrate light into the haptic experience, i.e., tangible light tangible interaction [2]. Research in this area studies how light itself may become more physically tangible, and how the interaction with tangible objects is influenced by light regarding, for example, visual attraction and gestural movements [3].

Light is an important means of information representation and feedback in HCI, and light-emitting interaction elements are omnipresent. Tangible interaction occurs through body gestures immediately [4] or emotional and conceptual awareness [5]. The interplay of light and tangible interaction connects user "context" and "presence", which supports an embodied and engaged interaction experience beyond haptic experience with a visual [6,7], emotional [8,9] and conceptual [10-13] awareness. For example, 
to turn on a lamp, we used to press a switch. However, in the context of tangible interaction with light, we can: (1) physically touch the lamp itself instead, (2) use an in-air hand gesture, (3) use other natural body behaviors, e.g., blink, blow, and face expression, and (4) use behavioral preference perception. In all the contexts, the lamp can sense and respond to users' control awareness, not as an "unfeeling" object. This is a valuable area to know: (1) how light is linked to the haptic experience, for example, playful interaction with light in the context of games [14]; (2) how the interplay between the two modalities creates an exceptional experience that cannot be achieved by any of them alone, where light enhances users' interactive experience.

It is good to have a comprehensive overview, however, we do not have such a research review yet. Therefore, we conducted a literature review of the studies of tangible interaction with light for the past 20 years. In this review study, tangible interaction with light contains two meanings: (1) light is an input modality, for example, use light to enter information or interact with a light beam to manipulate the HCI system; (2) light is an output modality to mediate information, for example, HCI is improved by light stimuli or feedback, which comes from ambient or discrete light information. Our method involves a systematic review and coding of 169 studies that were seriously conducted for exploring and displaying the research about tangible interaction with light. The results paint an in-depth picture of the research about interaction with tangible light in HCI.

\section{Research Questions and Contributions}

The review aims to understand the essence, process and result of how tangible interaction with light is conceptualized. In order to get a methodical and systematic understanding, we focus on the following four research questions in practices:

Q1: What makes tangible interaction with light an important research topic?

Q2: What are the characteristics of tangible interaction with light?

Q2.1: What are the research trends of tangible interaction with light over the years and countries?

Q2.2: What are suitable user interface mediums for tangible interaction with light?

Q2.3: What are known interaction designs for tangible interaction with light?

Q2.4: What are the functions of light for tangible interaction?

Q3: What are the conceptual research frameworks to guide the prototype design of tangible interaction with light, and how can the current research be classified?

Q4: What problems posed by the tangible interaction with light have been addressed or explored in current research? (Q4.1) What are the valuable research topics for the future? (Q4.2)

The main contribution is to summarize in a structured way the studies on tangible interaction with light of the last 20 years. According to our four research questions, we can see more specific contributions of our review work are: (1) to have a better understanding of the importance of tangible light research, which also contributes to a broader topic: tangible interaction; (2) to have a systematic knowledge about what their research trends, user interfaces, interaction ways, functions of light, and conceptual research frameworks. This information is helpful for HCI designers and researchers who want to know more details about the interplay between light and tangible interaction; (3) to show the problems addressed by tangible light research and provide valuable research topics for bringing tangible light closer to real-life application.

\section{Theory Background}

\subsection{Tangible and Embodied Interaction}

Embodiment should be seen as a fundamental concept for HCI [15]. Over the last years, research has established the exploration of this form of interaction-embodied interaction. Embodied interaction refers 
to the ability to involve one's physical body in interaction with technology in a natural way, such as by gestures [4]. The philosophical underpinnings of embodied interaction are widely accepted and it is closely related to the field of "tangible interaction" since embodied interaction transitions from the pure psychological interaction domain into the physical interaction space [5]. With the development of a range of technologies designed to sense movements of the body, embodied interaction is increasingly used in the design, analysis, and evaluation of the interaction with technology. However, there is still much work to be done to demonstrate their practical applications in HCI [16].

In order to work out what it means to design embodied technologies for embodied people in an embedded world, researchers are exploring it from the following four aspects. First, the turn to embodiment is a momentum in HCI, following the success of Dourish's [15] book "Where the Action Is". It is about understanding interaction in terms of practical engagement with the social and physical environment. Second, Klemmer, Hartmann, and Takayama [17] elaborated on bodily engagement with the physical and digital worlds from philosophical, psychological and sociological theory perspectives. Third, Hornecker and Buur [18] developed a tangible interaction framework that conceptualizes technologies about embodied interaction, tangible manipulation, physical representation of data and embeddedness in real space. At the same time, it offers four themes to help researchers understand the interaction with tangible interaction systems: tangible manipulation, spatial interaction, embodied facilitation and expressive representation. Finally, Hurtienne's [19] theory of embodied cognition is grounded in the ways of how people experience the world through physical interaction, and it emphasizes the value of using abstraction from specific contexts.

It should be noted that tangible interaction is an "umbrella term" used to describe HCI user interfaces and interaction approaches that emphasize [18]: (1) tangibility and materiality of the interface, (2) physical embodiment of data, (3) whole-body interaction, and (4) the embedding of the interface and the user interaction in real spaces and contexts. Tangible interaction presented a new direction for computer interfaces that brought computing back into the real world. Therefore, tangible user interfaces were envisioned as an alternative to graphical displays that would enrich user interaction. Normally, it was represented as to manipulate via physical interaction with tangible objects. The core idea was to allow users to do HCI by their bodies and to sense representation and control.

In all, to adopt tangible and embodied interaction means to have a particular sensibility and approach to view technology and design in the context of the world around us. An embodied interaction perspective takes full consideration of the ways human beings are embodied with both their perception and action. Thus, it allows us to view the interaction differently not as a split of perception and action. Different meanings are ascribed in interaction through our tangible and embodied interaction with the physical world and with each other. Rather than struggle to make sense of the inflexible meaning encoded in computers, we create and communicate the meaning of our actions by exploring, adapting and adopting interactive technologies, incorporating it into our world and everyday practices. For example, instead of using generic input devices such as a keyboard or mouse to control computers, we can use a meaningful natural body contact or gestures. This is a tangible and embodied interaction.

\subsection{Tangible Light Interaction}

Tangible Light Interaction (TLI) refers to an interactive visible light communication, which integrates intuitive ambient intelligence and social communication [2,3]. Human beings are the ultimate beneficiaries of TLI [20]. As we know, visible light communication is a new paradigm that could revolutionize wireless communication [1], which has been used in, for example, smart cities and human sensing. TLI is a unique research topic for tangible interaction, because light itself is not tangible. However, it is a good medium to augment the tangible and embodied interaction with visualized or sensible feedback. Particularly within the field of intelligent technologies, tangible light examples have shown how design concepts can support social interaction and remote communications in new ways [3]. 
TLI defines how interaction and behavior are mapped into light outputs [3,20]. An interaction could be an elaborated succession of activities defining for an embodied and emotional engagement. The user might be a person or many, which locally or remotely manage and control the interactive object. The aims of TLI could be to, for example, broadcast information to people, provide visual guiding, orchestrate a situation, create a particular mood, illuminate the room, display a graphic symbol or icon (still or moving), send data to a personal or group device [2,20]. For example, LightCube [20], a conceptual tool for learning contexts, could externalize students' emotional, motivational or cognitive states, and create appropriate ambient atmosphere correspondingly.

TLI contains two categories: (1) Light is a main and direct (not additional) feedback to tangible interaction [3]. Take turning a lamp on/off as an example, unlike pushing a switch, people can use hand gestures instead and even adjust its brightness. In such kind of situation, color, brightness and on/off changes of light is the direct feedback of a tangible interaction. (2) Light visualizes the results of tangible interaction [2]. Touching or moving a tangible object gives people a physical sense of tangible interaction, but light can augment it with a visual stimulation by light shining, color changing or character representing. For example, when playing a game has tangible objects, light can not only make people more engaged but also provide additional hint information.

\subsection{Theories in $\mathrm{HCI}$}

Theory works at an abstract level, enabling understandings and generalizations to be made about specific phenomena. Within HCI, a number of vehicles for disseminating these have been proposed, ranging from qualitative and descriptive concepts, themes, patterns, ideas, frameworks, to more formally and predictive, taxonomies, models and principles. They usually are based on assumptions and, at the highest level, aim to provide general laws, rules and formulas, which can be applied in a variety of contexts. Norman and Draper [21] have come close to articulating a general model of HCI, however, it is a conceptual model rather than a model in cognitive psychology or computer science. It is intended as a framework for understanding rather than as the content of such a framework.

An early example of using HCI theory was how to apply information processing theory to make predictions. Later, HCI moved on from its early roots in engineering, and started to mix science, engineering, art and design. Thus, the ways theory has been used and developed in HCI has become more diverse. For example, Bederson and Shneiderman [22] proposed five theories for HCI: descriptive, explanatory, predictive, prescriptive and generative, which form a generative circle with clarifying terminology, explicating relationships, testing hypotheses, and developing new theories. Rogers [23] added theories of informative, ethnographic, conceptual and critical to providing us different ways of understanding, conceptualizing and constructing arguments about user experience, interaction design and practice. Finally, Rogers [23] made Table 1 to provide an overall summary of the different roles theory has been developed for in HCI. They are not mutually exclusive and some will overlap to show the different ways that theory has and can play.

Table 1. A summary of the ways theory has been used and developed in Human-Computer Interaction (HCI).

\begin{tabular}{lll}
\hline Types & Description & Reference \\
\hline Descriptive & Clarifying terminology and guiding inquiry & {$[22,24]$} \\
Explanatory & Explicating relationships and process & {$[22]$} \\
Predictive & Testing hypotheses about user performance & {$[22,23]$} \\
Prescriptive & Providing guidance on how best to design and evaluate & {$[22,23]$} \\
Informative & Importing relevant findings to ground understanding of HCI & {$[23]$} \\
Ethnographic & Providing detailed descriptions arising from a field study & {$[23]$} \\
Conceptual & Eliciting frameworks for informing design and evaluation & {$[23]$} \\
Critical & Couching HCI in a cultural and aesthetic context & {$[23]$} \\
Wild & Developing new theories of technology use in situ & {$[22,23]$} \\
\hline
\end{tabular}




\section{Methodology}

Our review methodology consists of three main phases: planning the review, conducting the review, and reporting the results. After discussing the research purpose, we identified six search terms. They came from two considerations: (1) focused on the tangible, interactive, and directly involving aspects of the artifact: "tangible", and "gam*" (e.g., game or gamification); (2) related to the artifact's attribute of being a light source or including light in the interaction with the artifact, for example, "light", "lamp*", "bulb*", and "lantern*".

About the choice of the above search terms, two reasons need to be explained. First, "gam*" was one of the search terms, because game (gamification) is an important research area for tangible interaction, especially for learning and edutainment $[25,26]$. Second, we decided to not use "embodied" as a search term, because (1) "tangible" is a more accepted term in HCI, which is more closely linked to physical interactive artifacts such as prototypes, which we were interested in, and (2) we tried to search with the keywords of "embod" and "light", however, the related results were almost the same for the search with "tangible" and "light".

Before performing the search, the inclusion and exclusion criteria were defined. We include peer-reviewed papers, posters and book chapters with a conceptual or physical prototype published after the year 2000. However, we exclude non-English papers, dissertations and theses.

The search was conducted from December 2019 to April 2020 by the authors. The search uses two electronic databases: Web of Science (WoS) core collection and Association for Computing Machinery (ACM) digital library. WoS includes the main electronic databases for publishing papers about computer science, such as Engineering Village, IEEE, Springer, and Science Direct. The ACM is a comprehensive database covering computing and information technology, especially related conference papers. Thus, our search results are comprehensive.

Combining the main search terms and inclusion and exclusion criteria, as shown in Table 2, the search was conducted 24 times. The search combinations were with terms "tangible", "light*", "lamp*", "bulb*", "lantern", "gam*”, and "interact*”. The publication date was limited between 2000 and present. Total records identified through (1) WoS database search results are 8065 papers, (2) ACM are 1204 papers. In all, 9269 papers were retrieved. Because very general search keywords were adopted, we decided to screen the results with two strategies: first, read the paper title and abstract, and save the related results for the next step; second, read the paper content and get the final results for review. The final decision was based on whether it had a conceptual or physical prototype for tangible interaction with light or not. Finally, $169(1.8 \%)$ papers were studied and reviewed in our research.

The reasons for this high dropout rate (more than 98\%) are: (1) the WoS database has a very high number of unrelated papers. For example, using "interact* AND light*" as keywords in the title resulted in 3416 papers, however, more than $99 \%$ papers were engineering, instruments instrumentation, telecommunications, chemistry and so on. (2) Only the studies with a conceptual or physical prototype are included in our review, thus it further excludes some papers only with a conceptual idea (no prototype design diagram or sketch). 
Table 2. Search procedure and reviewed results (from year 2000 to present).

\begin{tabular}{|c|c|c|c|c|c|}
\hline Search ID & Title & Keyword & Abstract & Results & Review $^{\dagger}$ \\
\hline WOS-1 & & tangible AND light* & & 607 & 22 \\
\hline WOS-2 & & tangible AND lamp* & & 17 & 1 \\
\hline WOS-3 & & tangible AND bulb* & & 5 & 0 \\
\hline WOS-4 & & $\begin{array}{l}\text { tangible AND } \\
\text { lantern* }\end{array}$ & & 2 & 0 \\
\hline WOS-5 & gam $^{*}$ AND light* & & & 1217 & 3 \\
\hline WOS-6 & gam* AND lamp* $^{*}$ & & & 37 & 0 \\
\hline WOS-7 & gam* AND bulb* $^{*}$ & & & 47 & 0 \\
\hline WOS-8 & gam* AND lantern* $^{*}$ & & & 2 & 0 \\
\hline WOS-9 & interact* AND light* & & & 3416 & 12 \\
\hline WOS-10 & & interact* AND lamp* & & 2491 & 14 \\
\hline WOS-11 & & interact ${ }^{*}$ AND bulb* & & 94 & 0 \\
\hline WOS-12 & & $\begin{array}{l}\text { interact* AND } \\
\text { lantern* }\end{array}$ & & 130 & 1 \\
\hline ACM-1 & & & tangible AND light* & 330 & 72 \\
\hline ACM-2 & & & tangible AND lamp* & 25 & 5 \\
\hline ACM-3 & & & tangible AND bulb* & 63 & 7 \\
\hline ACM-4 & & & $\begin{array}{l}\text { tangible AND } \\
\text { lantern* }\end{array}$ & 2 & 0 \\
\hline ACM-5 & gam* AND light* & & & 50 & 0 \\
\hline ACM-6 & gam* AND lamp* $^{*}$ & & & 1 & 1 \\
\hline ACM-7 & gam* AND bulb* $^{*}$ & & & 19 & 0 \\
\hline ACM-8 & gam* AND lantern* & & & 0 & 0 \\
\hline ACM-9 & interact* AND light* & & & 367 & 29 \\
\hline ACM-10 & interact* AND lamp* & & & 12 & 2 \\
\hline ACM-11 & interact* AND bulb* & & & 334 & 0 \\
\hline ACM-12 & $\begin{array}{l}\text { interact* AND } \\
\text { lantern* }\end{array}$ & & & 1 & 0 \\
\hline Total & & & & 9269 & 169 \\
\hline
\end{tabular}

\section{Results and Discussion}

In order to response our research questions and make an in-depth analysis, the sections of "Results" and "Discussion" are put together.

\subsection{Importance of Tangible Interaction with Light (Q1)}

There are many perspectives to answer RQ1. However, we found the importance of tangible interaction with light came from three reasons: (1) it is a suitable approach for most diverse purposes; (2) it is used in different contexts; (3) it supports diverse user interactions.

\subsubsection{A Suitable Approach for Most Diverse Purposes}

Tangible interaction with light is a suitable approach for most diverse purposes such as education, games, and aesthetics. This shows us the possibilities of light in HCI, and also deepens our understandings of the relationships between light and tangible interaction and how light can be used for tangible interaction.

As shown in Table 3, the purpose of tangible light in the review results is investigated. Six purposes were summarized, which are for the controller (37 papers), education (24 papers), emotion (19 papers), aesthetics (26 papers), game (20 papers), and information visualization (33 papers). In addition, the other 10 studies were unknown for a specific purpose. 
Table 3. Purpose of tangible light in the review results.

\begin{tabular}{|c|c|c|c|c|c|c|c|}
\hline Purpose & Information Visualization & Controller $^{\dagger}$ & Aesthetics & Education & Game & Emotion Sensing & Others \\
\hline Number & 33 & 37 & 26 & 24 & 20 & 19 & 10 \\
\hline
\end{tabular}

As the most common purpose, the results of tangible interaction with light use light as a medium to visualize information, such as time [6,27], calendar events [28], temperature [29], alert [30], off-screen ambient display [31], text and image [32-35], real-time system status [7], gradual data [36-39], and health status [40]. The visualized information results from the tangible interaction and acts as a visualized feedback to users. For example, Hourglass [27] senses user's sleep and uses the lighted LED number to indicate the amount of sleep time. This means that the physical object is an exclusive presentation of the collected data of the user's sleep cycle and is using an ambient low resolution representation, which distinguishes it from screen interfaces. Metamorphic light [41] supports people to intuitively manipulate an image projected onto paper. Armura [42], a novel interactive on-body system, supporting both input and graphical output, such as moving the hand to different positions reveals what buildings lay in that direction. In LightBundle [43], the metaphoric plant properties lend themselves to interaction scenarios ranging from timing, location, social awareness to energy transfer. "I Like This Shirt" [44] explores the translation of social mechanisms in the virtual world into physical experiences with a shirt that responds to physical touches, which indicate "likes" and tracks and visualizes the "like count" in real-time on the shirt itself.

Tangible interaction with light has been designed as a new method to control devices, such as classic home lighting setups [45] or setups with shared control over lighting situations [46,47]. Projects within this category go beyond the traditional interaction with and control of lighting systems. Beside traditional interaction, such as pressing or flipping a lamp switch, these studies either have a natural body gesture interaction (e.g., in-air hand gesture) or provide a shared space for tangible interaction among several people. For example, it makes light manipulation a means to enhance the experience of the social or work-related environment. The specific control functions vary from turning on/off the lamp (e.g., [48]) to changing light color and brightness (e.g., [49,50]). In addition, tangible interaction with light provides a physical experience to control digital music [51]. For example, the adjustment of light brightness indicates music volume and light flashing represents music rhythm.

It is an emerging trend that tangible interaction with light adds some aesthetics design elements, such as culture [52], reflection [53], pragmatist philosophy [54], calm user interface [55], physical metaphor [56], media architecture [57], and olfactory sense [58]. For example, sounds of infinity [59], an interactive, low-resolution lighting display, portrays a magnified variation of the infinity mirror.

For education purposes, tangible interaction with light has shown to be a good ambient awareness tool for student feedback [60], group collaboration [61-65], peer connection [66], abstract concept displaying [10-13], and an interactive and cooperative play tool for learning [67,68]. For example, EnergyBugs [12] are energy harvesting wearable with features that invite children to move their bodies to generate tiny, yet usable amounts of electricity and illuminations with different light colors. Timelight [69], an ambient light display, the interaction with it helps children better understand the duration of time periods.

Games with light through animation and simulation have been designed for group communication [14], group games [70,71], drawing on the ground [72], multi-user music composer [73], gameboard [74], storytelling [75,76], exercise training [77-79], behavior change [80,81], and physical knowledge learning [82]. For example, D-TOX [81], a user-centered design to apply gamification to a smart lamp and mobile application reduces the frequency of smartphone usage during nighttime.

The user's emotion states can be collected and displayed with a lamp, which is created for emotional communication [83] and connection [9,84], a companion [85], and a positive effect among pedestrians [86]. For example, Angelini et al. [87] designed an anthropomorphic lamp that provides 
a novel and more natural way for the communication of five common emotions: happy, sad, trusty, ecstasy, and angry. Mole Messenger [88], a pair of connected creatures, helps children share and send messages to their loved ones who may be far away. Calmworklight [89], a desktop lamp, responds to changes in peoples' focus strengths captured by a commercially available EEG device.

Tangible light has shown some other possibilities, such as revealing how colored light indicates semantics in robotics [90], giving visual feedback about hand gestures without affecting phone on-screen content [91] and energy-saving lighting design [92].

\subsubsection{Used in Differentiate Contexts}

Tangible interaction with light has been used in different contexts, especially school and home. The exploration in such different contexts opens a door for interdisciplinary research and light has shown to be a good medium to improve interaction. As shown in Table 4, review results indicate school, outside places, home, and workplace are the four most common user contexts. In school, interaction with tangible light is used in the classroom to support student feedback, collaboration and conceptual understanding. Outside places, such as park [93], road [85], and building periphery [57], are installed with tangible light devices for playing, interaction and decoration. At home, tangible light is an important component for a smart home as a smart lamp [45,48,49], clock [43], and new smart device [8,81]. In the workspace, tangible light is used for danger alarm [30] and interactive office lighting [47]. The other 86 studies had no specific user contexts, but instead generally explored design concepts such as emotion and aesthetics.

Table 4. User context of tangible light in the review results.

\begin{tabular}{llllll}
\hline Context & School & Outside Place & Home & Workspace & Others \\
\hline Number & 27 & 23 & 21 & 12 & 86 \\
\hline
\end{tabular}

\subsubsection{Support Diverse User Interactions}

As shown in Table 5, for interaction design, most studies (114 studies) allow only one user to interact with the tangible light. However, there are 12 studies for two users and 27 for three and more participants. These studies indicate that light is good at improving user's remote tangible interaction experience, which is beyond the concept of tangibles and implies a requirement of emotional connections. This can be an important research direction for how HCI improves human being's connections and relationships. In the review results, most two-user studies are to create interpersonal awareness and communication between two people in a remote distance, such as SnowGlobe [8], LumiTouch [94], SmallConnection [83], touch trace mirror [84], connected candles [9], and Mole Messenger [88]. Interaction among three or more users is designed for cooperative play $[14,68]$, collaborative problem solving [62] and shared interaction [65] with light.

Table 5. Numbers of interaction users with tangible light in the review results.

\begin{tabular}{lllll}
\hline Interaction User & One & Two & Three or More & Unknown \\
\hline Number & 114 & 12 & 27 & 16 \\
\hline
\end{tabular}

\subsection{Characteristics of Tangible Interaction with Light (Q2)}

\subsubsection{Research Trends of Tangible Interaction with Light (Q2.1)}

For all the selected 169 review results, there are 147 conference papers, 21 journal papers (includes one review paper [95] about user interaction with everyday lighting systems), and one book chapter. As shown in Figure 1, 88.8\% of papers are published between 2010 and 2019. Particularly for the past five years from 2015 to 2019, each year's publication is above 15 papers. It means that, in general, studies of tangible light is increasing. 
As shown in Table 6, the review results come from 27 countries. The USA, Netherlands and Germany have the highest number of published papers, which are 33, 25 and 20, respectively. Subsequently, Korea (13), Japan (12) and China (11) have a similar number of papers. Many reasons lead to this distribution. However, one not to be ignored is that there are some good HCI research groups in these countries, such as the tangible media group in MIT (USA), the Human-Technology Interaction group from Eindhoven University of Technology (Netherlands), media informatics at the University of Munich (Germany), the intelligence and interaction center at the Korea Institute of Science and Technology (Korea), Human-Computer Interaction research communities in the University of Tokyo (Japan) and the IDEA Lab at Zhejiang University (China). In contrast, eight countries such as Belgium and India have only one related paper.

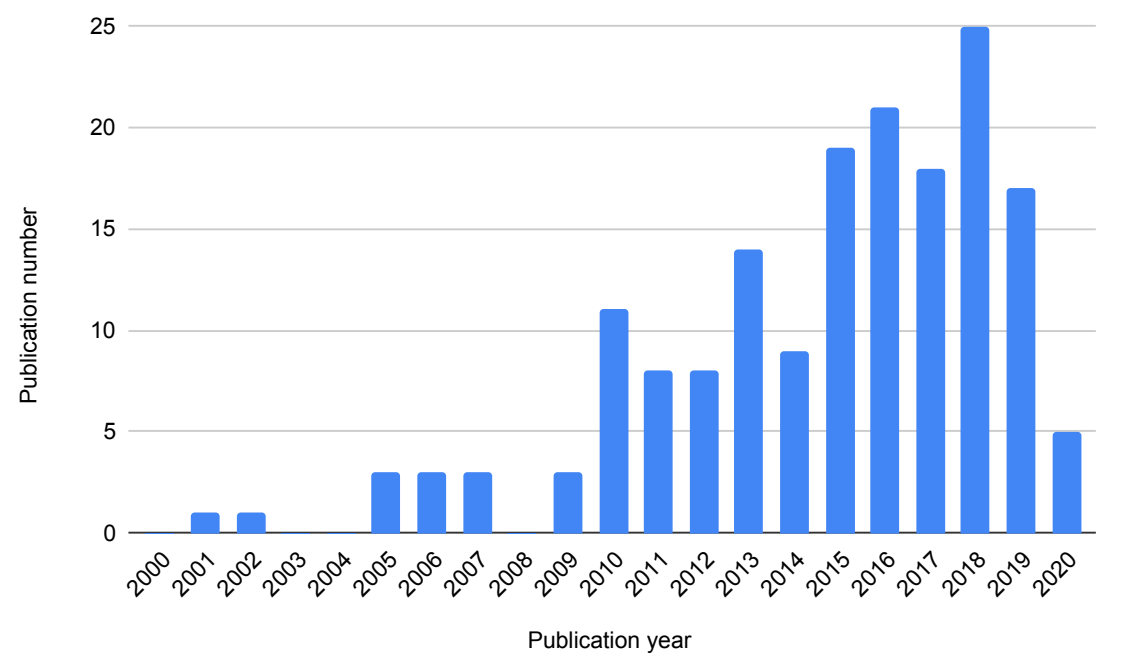

Figure 1. Publication year distribution of review results.

Table 6. Country distribution of review results.

\begin{tabular}{llllll}
\hline Country & Number & Country & Number & Country & Number \\
\hline Australia & 6 & India & 1 & Poland & 1 \\
Austria & 2 & Iran & 1 & Portugal & 2 \\
Belgium & 1 & Italy & 4 & Russia & 1 \\
Canada & 5 & Japan & 12 & Singapore & 2 \\
China (Taiwan) & $11(5)$ & Korea & 13 & Sweden & 3 \\
Denmark & 3 & Luxembourg & 1 & Switzerland & 2 \\
Finland & 3 & Mexico & 3 & Turkey & 1 \\
France & 4 & Netherlands & 25 & United Kingdom & 7 \\
Germany & 20 & New Zealand & 1 & USA & 33 \\
\hline
\end{tabular}

Note: One paper's country is unknown.

\subsubsection{User Interface Medium with Tangible Light (Q2.2)}

The user interface is the space where the interaction between humans and tangible objects occurs and in the case of tangible interaction it is literally the touch point. Generally, the goal of user interface design is to produce a user interface that makes it easy, efficient, and enjoyable (user-friendly) to operate a machine in a way that produces the desired result. We investigated the user interface medium with tangible light in the review results. As shown in Table 7, user interface medium with tangible light in the review results includes 14 kinds. The most common medium is LED objects, which are small light resources that are inserted or combined flexibly with the real life objects to show HCI results. Unlike functional objects, for example, lamp and dice, it refers to independent LEDs integrated for tangible interaction. For example, Chhikara and Hespanhol [75] made 11 boxes 
equipped with LED strips and pressure sensors. Once the sensors in one box are activated, they trigger the LEDs in the next box to light up.

Table 7. User interface medium with tangible light in the review results.

\begin{tabular}{ll}
\hline Medium & Number \\
\hline LED Object, e.g., hourglass, LED box, object with LED-edge & 38 \\
Lamp & 31 \\
Interactive System, e.g., game system, multimodal interactive system & 23 \\
Interactive Object, e.g., virtual lamp, robot, toy, exercise device, clock & 25 \\
Cube, e.g., music cube, calendar cube, cube toy & 9 \\
Smartphone & 8 \\
Soft Material, e.g., elastic ball, inflatable balloon & 7 \\
Light Switch & 6 \\
Clothes & 6 \\
Paper & 4 \\
Mirror & 3 \\
Hand and Foot & 2 \\
Bracelet and Jewelry & 3 \\
Plant & 1 \\
\hline
\end{tabular}

Note: There are 3 papers that are inclusive in Table 7, because they are summary studies and include many tangible object descriptions.

Subsequently, lamps are used for lighting but often designed to do a tangible interaction. Many good innovative concepts and examples would subvert our original perception to HCI, such as keyboard and mouse. Kao et al. [43] designed a lamp named LightBundle to use natural gestures (e.g., binding, peeling, pointing, twisting, and grasping) to interact. These interactive ideas were inspired from the observations of interactions with plants. D-TOX [81], a lamp as well, the color and intensity of which vary depending on the smartphone usage, aim to give the user a visualized feedback. CREATUREs [96], an interactive interior lamp, could recognize a user touching lamp-shades. When it senses the shadow, the lamp-shade will fill out and the light slowly flickers. Alternatively, when it detects sounds or voices nearby, it flickers fast.

Interactive system means a scene that is created to make users collaborate and engage in the context of tangible light, which includes more than one tangible object. For example, Thrii [97] is a system to allow three participants to manipulate a large spherical object of which the movement is tracked via an embedded accelerometer. An analysis engine computes the similarity of movement for each possible pair of objects, as well as self-similarity (e.g., repetition of movement over time) for each object. COMB [98], a three tangible modular interface, enables children to practice electronic or digital music with natural and intuitive behaviors. Interactive objects refers to the interaction medium with light, for example, Miller et al. [34] made an experiment to ask people to move a tangible object from a start zone to one of four equidistant colored zones with light. A word representing a color would be illuminated with colored lights on the table.

The cube is a convenient object to get a physical experience of tangible interaction. The biggest advantage of the cube is that it has six sides and has been used to design different interactive modes with light. For example, we can control digitally stored music on a computer by means of gestures and positioning with MusicCube [51] and AudioCubes [99]. With the most common use of smartphones in our daily life, smartphones with light can be very helpful in some situations. For instance, light allows mobile devices to visualize off-screen objects by illuminating the background without compromising valuable display space [100].

Soft material refers to the tangible light user interface designed with an elastic ball [36], an inflatable balloon [101], a cushion, etc. Take Sugiura et al.'s [73] music instrument study as an example, two sensors were embedded in either side of the cushion and it can detect the touch position and applied pressure. Keys are shown in different colors (projected) and the pitch of each key 
can be changed using the amount of pressure applied. Multiple cushions can be combined to make a multi-user music composer application. Light switch means the interaction process is not through light itself but with a switch. For instance, Czuszynski and Ruminski [102] designed a contactless, hand gestures-based control of a switch with a gesture sensor to control the light.

Wearable light objects embedded in the clothes have been used in two ways. First, it explores the translation of lightweight social interactions into the physical world with visualized information, for example, Najafizadeh et al. [44] designed a shirt that can respond to physical touches, which indicate "likes" and tracks and visualizes the "like count" in real-time on the shirt itself. Second, clothes using light can be a considerable element of the emotion expression. For example, the brightness and colors of the light shown in the clothes have been used to visualize people's emotion statues [103], which means light helps people exchange feelings. Paper using light to show information gives people a virtual experience to interact with the text or image. For instance, the LampTop [104] enables an effective low cost touch interface utilizing only a single camera and a pico projector, and people directly use fingers to interact with the image.

Mirror is designed to receive a message and you place a finger on the emerging light on the mirror's surface [84]. Hand [42] and foot [105] are a novel on-body interface with light and the interaction supports both input information and graphical output. Light with bracelet [106] and jewelry [107] is designed to have modular wrist-worn digital jewelry for multiple purposes. In addition, there is only one research about the tangible interaction between plants and light, but it is an innovative research project. Sonnengarten [108] is a media art installation based on human-plant interaction, which aimed to increase people's awareness of nature via a light-based reaction to touching the plants.

User interface medium of tangible light in the review results is unconventional and diverse. In this review study, the user interface is the means by which users interact with a tangible light prototype to accomplish some purpose. A great deal of literature in HCI discusses user-centered design, which means we study user behavior and design an interface to afford it. However, the tangible light review results show a converse design concept: create a new experience for the users. As a universal element in our daily life, light can create an unexpected integration into other materials.

There are some unconventional and creative user interface design ideas in these studies. As we know, user interface is a connection point for interaction and communication between users and designed devices. Usually, a user interface means a display or a touchable screen, a keyboard or an instrument, a mouse or a controller and the explanation of interactive instructions. Possibly, it is also the way through which a user interacts with an application or a website. However, tangible light research shows us many unexpected examples of user interface mediums, such as plants, clothes, mirror, paper, and soft material. They provide valuable possibilities of future research about tangible interaction design. For example, soft material opens a door to broader interaction forms other than lamps, light switches, and LED objects; clothes decorated or embedded with light can be both a practical purpose (e.g., safety) and an aesthetic artwork; paper and mirror, which are interfaces to show information by light, enrich our daily life with a sense of embodiment. It is closer to our natural behavior and easier to understand. Finally, as a symbol of the natural world, the plant user interface makes the $\mathrm{HCI}$ interesting and thought-provoking. An appropriate $\mathrm{HCI}$ design might make people communicate with plants, for example, to know plant requirements of water, light and chemical materials, and in contrast, let plants indicate people's living environment.

The diversities of user interface designs in the tangible light research give us a whole picture of design possibilities in HCI. Besides a traditional interface medium (e.g., LED object), we have seen 13 other interface designs. It ranges from perceptually theme-related objects (lamp and light switch) to unusual interactive mediums such as soft materials and the human body. This transformation has brought tangible light to a broader application, such as gamification, augmented reality, fashion design, and people-plant interactions. The research about the above areas is only a few in the review results, but they are all shown as a good example to show the possibilities of tangible light in new ways. 


\subsubsection{Interaction Behavior with Tangible Light (Q2.3)}

HCI studies the ways human interacts with computer technology in their work and activities. The term "interaction" in the HCI nowadays contains most technology from obvious computers with screens and keyboards to mobile phones, household appliances, in-car navigation systems and even embedded sensors and actuators such as automatic lighting. As shown in Figure 2, we summarized and classified all the types of interaction behavior with tangible light in the review results. It contains two components: direct interaction (hold, tap, press, shake, touch, move, squeeze, body interaction and multiple modes) with 104 (61.5\%) cases and indirect interaction (in-air hand interaction and inductive interaction) with $65(38.5 \%)$ cases.

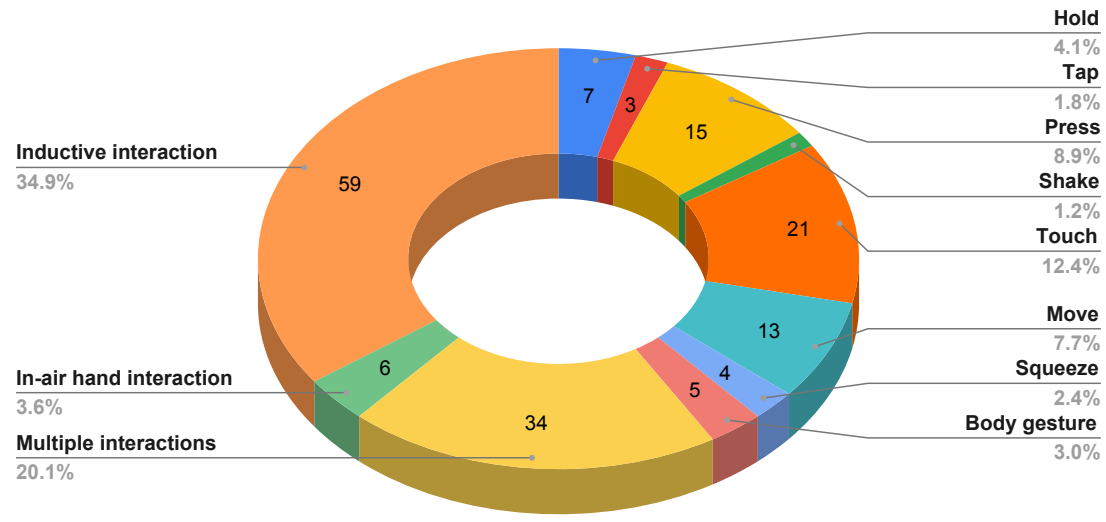

Figure 2. Interaction with tangible light in the review results.

Among the nine direct interaction behaviors, multiple interactions (34 studies) and touch (21 studies) have the most cases. In contrast, shake (2 studies) and tap (3 studies) have the least cases. Multiple interactions refer to the prototype as having more than one way to interact. For example, LightBundle [43] has the hold, grasp, pick and twist interaction designs for different scenarios. Lantern [62] has turning interaction for users to choose an exercise and press interaction for help seeking.

As a common interaction form, touch has more than four times the amount of studies than body gesture, squeeze, tap and shake. Body gesture in this review means the interaction by user's mouth [56,87], foot [105], and expression [109] and so on. Squeeze interaction is suitable for soft materials, for example, cushions for playing piano [73], soft objects for games [39] and squeeze box [14] for lightening up the workplace through playful and lightweight interactions.

The shake interaction needs to move the objects quickly, for example, shaking ColorFlare [63] to make the light start blinking and transmit its color to another ColorFlare. SnowGlobe [8], by shaking one's globe will cause the other person's Snow Globe to light up brightly. In contrast, the move interaction needs to move the objects slowly. For example, Boda blocks [64], which is a kit with a set of 16 luminescent cubes that can be arranged in a variety of configurations and programmes. A tangible user interface designed by Seaborn and Antle [110], when the user is close to the correct position, the temperature increases and red light slowly appears; when the user moves away from the correct position, the temperature drops and the red light dims to blue.

Tap interaction refers to touching the objects gently two times. For example, the expressive point lights [111], users can tap it to explore the expressiveness designs with the point lights embedded on ubiquitous devices. Press interaction needs pressure to be applied to the objects, for example, Plux [49], the brightness and saturation of which are determined by the user through the amount of pressure applied when pressing the tangible Plux controller. With Lantern [62], users can press it to call for 
teacher's help in the classroom. With Mole Messenger [88], each Mole Messenger box houses a pushable mole. When one is pushed, the other pops up, as if traversing to the other side of the globe. Finally, hold interaction allows users to hold the prototype in hand. For instance, with NeonDough [112], by holding and closing two NeonDoughs, the lights inside can show different colors based on their resistance values.

For the indirect interaction, it mainly has two categories: inductive interaction and in-air hand interaction. Inductive interaction refers to the interactions without direct physical contact and the system can sense the user's behavior. Take Calmworklight [89] as an example, the lamp's expression depends solely on the user's level of focus. If it is strong, the lamp returns a steady, calming warm light. If not, the light emission follows a breathing pattern, aiming to encourage the user to be more mindful of their breath and to cultivate a meditative focus. In-air hand interaction means no physical touch with the objects, but instead the device recognizes the user's hand gesture via, for example, cameras or sensors. It is an inductive interaction, but we made a separate category, because: (1) "hand" is the most commonly used for tangible interaction; (2) "in-air hand interaction" is an important branch of "gesture interaction" research. This category is convenient for people who are only interested in "gesture interaction" to know the situation; (3) it is a contrast with the physical hand interaction, for example, "hold", "tap", "press". For example, Lumigami [48], turning on, turning off or adjusting its brightness, the user needs to place his hand below this lamp and follow some design rules. "Tangible Lights" [45], an in-air gestural control of home lighting, has explored many gestural interaction styles for lighting control.

Interaction behavior in the review results is both embodied as well inductive. Interactive behavior defines how users connect and respond to the objects, which is largely determined by different interactive designs. When we interact with an object, some questions automatically come to our mind, such as what it does, how it works, and what operations are possible. Even a very subtle difference of operation gesture designs will produce an obvious different psychological perception. In designing for tangible interaction, people think about how to involve hands, eyes, and other physical aspects of the human body in the interaction. This supplements the pure cognitive behavior and includes the user's mind and body as they potentiate each other to create a more rich user experience.

More than $60 \%$ of research in the review results is about direct interaction that needs the users' physical body to interact with the light in a natural way. First, hand gestures, such as hold, tap, press, shake, touch, move, and squeeze, summarized from the review results, move users' interaction off the screen and into a real world. Therefore, we understand the world and ourselves in a physical and social world with embodied factors. This perspective transformation produced many innovative designs for the benefits of human, for example, emotional communication. Second, body gestures (e.g., foot and mouth) are beyond human's innate traits of being able to manipulate with our hands. It provides a more engaged and embodied user experience of a light artifact. Finally, more than one embodied interactions collaborate and communicate and make meaning through physically light objects in the real world. It shows how to involve hands, feet, mouth and other physical aspects of the human body in the interaction.

Inductive interaction, such as in-air hand interaction, portrays the possibilities of interacting without user's physical contact, such as eyes and face expression. The tangible light objects contain some kind of sense and computation. Even though the inductive interaction is designed without physical and tactile perception, users can feel and manipulate the tangible light object. For example, vision augments user's information acquisition and understanding with the variabilities of light characteristics. Face expression perception, which is through capturing with computer sense and displaying with light, is an obvious intangible interaction, but reflects the user's deep emotion. This interactive process is an integration of mind (thought) and behavior. In the future, embodied and inductive interaction can complement each other and create a more inclusive interaction experience for users. 


\subsubsection{Light Function for Tangible Interaction (Q2.4)}

Input and output are two interactive channels for HCI. We investigated the functions of light for the tangible interaction in the review results. As shown in Table 8, most studies have light as outputs of tangible interaction, which is good to improve feedback experience.

Table 8. Classification and examples of light as input/output in the review results.

\begin{tabular}{lll}
\hline Input/Output & Number of Results & Good Examples \\
\hline Input & 7 & Touch trace mirror [84], Thrii [97], interactive tangible game [77], onNote [113] \\
Output & 159 & MusicCube [51], Armura [42] \\
\hline
\end{tabular}

Note: There are 3 papers that are inclusive in Table 8 , because they are summary studies and include many tangible object descriptions.

As an input for tangible interaction, light is mainly used for indicating to users how to do a tangible interaction to get information, for example, messages from remote friends, navigation information, and sound. For example, Touch trace mirror [84] uses light to guide the user to move his or her finger on the mirror to understand the messages from a remote friend. Thrii [97] uses light to indicate to users how to adjust gestures to reach a similarity of movement. An interactive tangible game [77] is used for the training of post-stroke reaching, where light lights up to guide users to move their hands. onNote [113] allows playing printed music scores as a musical instrument, where the user follows the light indicated with a red circle.

As an output for tangible interaction, light is either ambient information or results of tangible interaction. For example, Armura [42] supports hand gesture input and graphical output with light to read information. "I Like This Shirt" responds to physical touches, which indicate "likes" and tracks and visualizes the "like count" in real-time on the shirt itself [44].

\subsection{Conceptual Framework for the Design of Tangible Interaction with Light (Q3)}

Referring to the nine HCI theories summarized in Table 1, we coded the 169 review results about how they design and conduct the tangible light studies. As shown in Figure 3, the most frequent theories used and developed within these studies are explanatory (47 studies) and critical (36 studies). The least research is about conceptual (none study) and descriptive (1 study) theories.

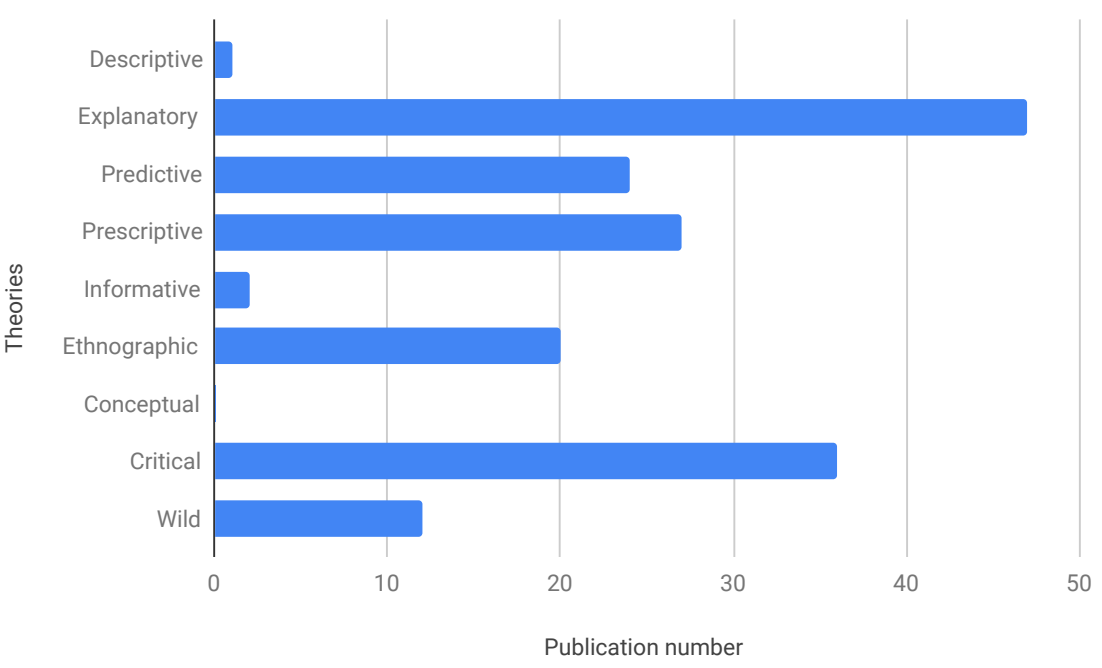

Figure 3. Conceptual framework used in the review results.

For the 47 studies portrayed by explanatory theory, the development processes of the tangible light prototype were explained. Specifically, it is a process with user study, conceptual design, 
prototype development, and prototype test. For example, Thrii [97], which is a multimodal interactive installation that explores levels of movement similarity among its participants, has four prototype development sections: previous work research, tangible sphere design, results analysis, and feedback improvement. Explanation of the design and application process benefits peer researchers. Within all the review results, critical theory supports 36 emotional, aesthetic and cultural tangible light studies. For instance, Angelini et al. [87] introduced a novel and more natural way for the communication of emotions with the ADA lamp. The whole design process was conducted with the purpose of creating an interactive experience with systems for the computer-mediated communication of emotions through ambient displays.

Since tangible interaction with light is a familiar but fresh research topic, it is good to see more than $20 \%$ of studies have been conducted to explore its application in a new context, such as emotion, aesthetics and culture. When the research purpose focuses more on creating new, creative, conceptual ideas, it helps to develop new theories of technology use in HCI. In the review results, only around $7 \%$ of studies are wild theory oriented, but they provide us good examples of how to design a concept idea or conduct an experiment of tangible interaction with light. Thus, in order to enrich this research topic, we should be more oriented by critical and wild theories. The reasons we have mentioned: (1) light itself is intangible, but has good attributes (e.g., color, brightness, information visualization) to facilitate the tangible interaction. Without an innovative theory, i.e., wild theory, to guide the conduct of the research, it would be hard to elaborate the research frameworks and values; (2) light is everywhere and so common to think of as research. If following a normal framework to design a study of tangible interaction with light, it might be difficult to find valuable research innovations.

The whole tangible light research in the review results shows a tendency from guided by explanatory and prescriptive theory to wild theory. As a new research paradigm, wild theory is useful in advancing knowledge in the HCI field. It is a part of a design discourse rather than formulated into a specific prediction or explanatory framework. Meanwhile, it implies a shift in design thinking: instead of developing solutions that fit in with existing practices, tangible light researchers experiment with new technological possibilities that could change user's behaviors, i.e., research through the design process. It informs research, generates insights based on the direct involvement with the prototype, and reveals challenges and opportunities. An applied field like tangible light research is to think how to best use the wild theory and follow the basic ideas: create an interactive opportunity, do an experimental intervention, and encourage a different behavior. Normally, this different behavior comes from a new and innovative design concept.

Tangible light, as a universal interactive technology, has provided good examples to innovate users' interactions, change their behaviors and visualize their emotions. They are valuable directions influenced and guided with wild theory. As an example of refining user interaction [8,9], tangible light improves the communication between people in remote places. With an interpersonal awareness design, such as a tangible movement or touch sense, it gives remote people a connected experience. In addition, beyond a normal lightning device, some tangible light research has been designed to regulate user's behaviors, for example, sleeping time, smartphone usage time, and exercise tracking. Emotion perception and visualization with tangible light brings a new research area to integrate behavior psychology and HCI. It transfers traditional visible interaction into a latent and subconscious interaction. Currently, it is a new area that does not have enough findings yet and requires more research.

Findings of tangible light research can be used to understand and shape new technology interventions in our daily living. They contextualize technologies broadly in HCI. For example, instead of designing research to make a prediction and apply it to a specific problem, tangible light research has been developed to address more fully the interdependencies among design, technology, behavior and art. We can view this hybrid form of innovative design-technology-augmented artistic reality-as engaging in an artistic space with embodied technologies. The focus is on how to augment, 
facilitate, or change the user's experience in ways that individuals naturally have. A number of intersections between the space, behavior, technology and cognition could be further explored.

\subsection{Problems Addressed by Tangible Light (Q4.1)}

We summarized the problems tackled by tangible light in the review results. In all, six problems are addressed: weak interaction (81 papers), do not know how to interact (30 papers), interaction lacks innovation (35 papers), collaborative interaction ( 9 papers), remote tangible interaction ( 7 papers) and emotional interaction (4 papers). In addition, three papers are summary studies and, therefore, not counted in the above categories. In order to see how tangible light fits these problems, Table 9 was made.

Table 9. Problems addressed by tangible light.

\begin{tabular}{ll}
\hline Problems addressed in the Review Results & Advantages of Tangible Light \\
\hline Weak interaction (e.g., feedback and process) & Visualize feedback and create an ambient environment \\
Do not know how to interact & Show interactive hints \\
Interaction lacks innovation (e.g., aesthetics) & Light properties (e.g., color and brightness) and characteristic \\
& (e.g., ambient and visual) and characteristic (e.g., ambient and visual) \\
Collaborative interaction & Create collaborative environment \\
Remote tangible interaction & Ambient atmosphere and tangible experience \\
Emotional interaction & Light atmosphere \\
\hline
\end{tabular}

\subsubsection{Weak Interaction}

Digital and intelligent devices are becoming common in our daily life. Light is a good ambient condition to embed on ubiquitous devices. However, these "smart" devices seldom provide sufficient communications to help users have situated awareness of the surrounding status. Tangible light explored the expressive designs of how light can be embedded on ubiquitous devices. For example, Liu et al. [111] demonstrated how designers could develop different light patterns and compose them with performative arrangements to convey informative clues about what is happening within the system. In all, light is useful for showing informative feedback. Tangible light takes advantage of tangible experience and continues feedback, which makes the interaction experience become more engagable.

Compared to traditional HCI, tangible interaction is a more engaged interaction. However, without elements, for example, light, sound and tactile, it is hard to have a real sense of tangibles. Light is a good element to improve the interaction process. For example, Sounds of Infinity [59] is an interactive, low-resolution lighting display that portrays a magnified variation of the infinity mirror. Developed for an outdoor light and music festival, the installation provides a retro-futuristic experience for audiences and explores how playful interactions might impact the behavior of people in public spaces.

\subsubsection{Do not Know How to Interact}

Tangible light is useful for increasing users' situated awareness and guiding them on deciding how to interact without overburdening. TUIs provide a digitally-augmented physical space for interaction. However, people without technology knowledge (e.g., children and old people) would have trouble knowing how to interact. They need more intuitive designs and light is a good medium to teach them how to interact. For example, Yang et al. [78] designed an upper limb training device for strengthening the coordination of the upper limb. Patients glide the device on a color map and reach a certain color point according to LED instruction. Successful reaching would trigger both visual and auditory feedback. For the problem of not knowing how to interact, light provides hint information. Moreover, it integrates into all the interaction processes, which other material could not replace. 


\subsubsection{Interaction Lacks Innovation}

New and innovative tangible interactions are an important research direction in HCI. Light has inspired many innovations for tangible interactions, because it is an intangible medium to do a tangible interaction. For example, LightBundle [43], a metaphoric plant, has properties for interaction scenarios ranging from timing, location, social awareness to energy transfer. It inspires from plant's properties to design a grasping light idea. Shi et al. [56] designed a manipulated light to take advantage of natural physical metaphor (waving, clapping and blowing) to achieve a heightened legibility and seamlessness of interaction between people and information.

New technologies provide expanded opportunities for interaction design. The growing number of possible ways to interact, in turn, creates a new responsibility for designers: Except TUI prototypes' visual aesthetics, one has to make choices about the aesthetics of interaction. This issue recently gained interest in HCI research [114]. Light has good properties, for example, color and brightness, to improve the feelings and meanings an interaction is enveloped in rather than the interaction results. For example, Ying et al. [52] designed an interactive lamp, which used a cultural design method on multi-sensor technology design. The purpose of this work is to integrate culture with technological industrial design.

\subsubsection{Collaborative Interaction}

There are many tangible interaction studies about individuals, not so many for the group level [18]. However, tangible interaction is good to provide a collaborative and entertaining learning experience. For example, TouchTomb [115], a situated tangible gamification installation, has enhanced informal cultural learning for young visitors and fostered engagement and collaboration among them. Tang-Phys and Tang-Dix [116], interactive prototype museum installations, are suitable for tacit heritage knowledge's embodiment, physical abstraction and materiality.

Light has shown to be a good ambient for collaborative interaction. In the review results, there are some good examples, such as Thrii is a multimodal interactive installation that explores levels of movement similarity among its participants [97]. Game of Light [71], a $6 \times 6$ arrangement of 36 squares for group interaction. To join the game, the user hangs their completed lantern in the gallery grid. After placement, the lantern begins to glow and interact with its neighbors-showing a changing pattern of light.

\subsubsection{Remote Tangible Interaction}

More than 20 years ago, tangible interfaces were being used for remote collaboration and communication [117]. At the beginning, it used a concept called Synchronized Distributed Physical Objects to create an illusion that remote users were interacting with shared physical objects [117]. Its applications include Illuminating Light (a Luminous-Tangible Interface for holography simulation) [118], PSyBench (allowed movement on one board to be reproduced on the other board) [117] and inTouch (two people separated by distance can feel the other person's manipulation of the rollers) [119]. However, this approach suffers from three disadvantages [120]: (1) interface graphics could only partially simulate the sense of co-presence; (2) remote users are not physically embodied; (3) object movement resulted in a disconnected experience.

To extend the physical embodiment of remote participants, a new idea named physical telepresence was proposed [120]. It added telepresence robotics [121] to the physical embodiment of the shared content, in which shape displays could physically embody remote users and objects to enable communication and collaboration. It has an advantage of increasing the user sense of presence by capturing and rendering shapes, which extend human capabilities with computer-mediated interaction techniques. However, it requires a special telemanipulation setup, which means it is hard to be used the daily life.

There are many forms of communication that aim to ease the feeling of longing for distant loved ones but they do not enable people to feel physically connected to one another. Tangible light has 
the advantage of integrating remote interaction and atheistic. For example, Connected Candles [9], a peripheral aesthetic display has been used to create awareness and connect people in long distance relationships. Light is a sign of connection, not only physically but also emotionally.

\subsubsection{Emotional Interaction}

With new developments in TUI and emotion sensing technologies, it is a new research direction to develop TUI that can infer emotional well-being of human beings. People will be accustomed to interacting by tactile stimuli as touch is critical for physical and mental well-being. At the same time, light is a good interactive feedback to stimulate people's deeper emotions. For example, Inter-glow is a system that facilitates close interaction and communication among users in real spaces by using multiplexed visible-light communication technology [122].

\subsection{Valuable Tangible Light Research Topics for the Future (Q4.2)}

Tangible light studies in the review results have shown us a multitude of perspectives, concepts, and approaches regarding how to benefit from tangible light. The analysis and discussion of tangible light's research trend, light function, user interface medium, interaction behavior, and conceptual frameworks revealed four important research experiences for the future:

First, light as an input for tangible interaction has many possibilities to investigate. As we have known, studies about tangible light are increasing, which might be influenced by the rise of tangible interaction research. It is easy to understand light can be used for visual feedback of tangible interaction, where light is an interaction output. However, light can also be an input in HCI. The relationship between light and tangible interaction, i.e., light is a reason or result of interaction, contains many possibilities for innovation. How light, which is intangible, can be used as an interactive medium for tangible interaction? How can we manipulate the physical objects with light? If we look at what happens for the interaction between light and the physical world, we can observe phenomenons such as casting shadows, light reflections, transferring heat, and creating emotions. However, our review revealed that light as an input modality remains almost unconsidered.

Second, researchers should leave the lab to find and investigate HCI ideas and designs in our daily life. Wild theory encourages new and innovative ideas, which suits tangible light research very well. It is important to build functional prototypes to enable an in situ experience but use new interaction concepts. Light is common but easy to be ignored when thinking of an element for HCI. Thus, new ideas become more important, which are easier to get from the observation and experience in the environment around us.

Finally, tangible light studies have considered aesthetic, emotional or cultural needs, which are easy to be ignored but an important innovation. Traditional design ideas often come from existing and practical problems. Their aims are to solve and improve user experience. For example, unlike presenting information only with digital screens, the ambient feedback method has become a good option. However, this requires designers and researchers to rethink new ways for information representation, which is beyond text and picture. We need a more open and exploratory research process, which is driven by the purpose of generating knowledge and ideas. When trying to solve a problem, do not ignore the insights acquired from the design process.

In all, we see the potentials for the research community to further explore and investigate tangible light designs. We can ask ourselves questions about: how to involve light as an intangible medium for tangible interaction designs, how can we have a better understanding of human-light interaction, and how can we make light a unique interaction experience in $\mathrm{HCI}$ ?

\section{Conclusions}

We compiled a systematic literature review of 169 tangible light studies from the last 20 years of HCI research. The demographic overview of the review results shows an increase in publications regarding this topic over the years originating from all over the world. Further, we discovered that 
tangible light has been used for diverse purposes and in many user contexts. This diversity-in the opinion of the authors-shows one of the advantages of tangible light: comprehensibility and familiarity, which are due to its common interaction and feedback modalities. We as human beings interact with the world around us mainly through haptic manipulation and understanding, tangible light integrates seamlessly into our living environment sensual experience.

It is helpful to do an analysis and discussion about what makes tangible light important and what problems have been addressed. Our insights might not be comprehensive, but elaborate some real problems for tangible interaction with light: weak interaction, do not know how to interact, interaction lacks innovation, collaborative interaction, remote tangible interaction, emotional interaction. Not all the problems are unique for tangible light research, but some have been seen in a broader area tangible interaction as well. However, tangible light research is still meaningful, because it is a suitable approach for most diverse purposes, has been used in different contexts, and supports diverse user interactions. Moreover, it inspires us to think of the possibilities of light as an input for tangible interaction and how to consider aesthetic, emotional or cultural needs for HCI.

Overall, even though we might lack a very comprehensive analysis and discussion, we presented an in-depth overview of tangible light studies. We see our work is a fundamental resource to inform, inspire, and motivate new research in this field. Light not only helps us to transfer from a discrete to a more ambient and peripheral presence of technology in our everyday environment, but also involves-in combination with tangible interaction paradigms-our whole body and mind.

Author Contributions: Conceptualization, Y.L., B.R. and H.H.; methodology, Y.L. and H.H.; investigation, Y.L.; writing—original draft preparation, Y.L.; writing—review and editing, B.R. and H.H.; supervision, H.H.; All authors have read and agreed to the published version of the manuscript.

Funding: Authors are supported by the Elite Network of Bavaria (Project number: K-GS-2012-209).

Conflicts of Interest: The authors declare no conflict of interest.

\section{References}

1. Rehman, S.U.; Ullah, S.; Chong, P.H.J.; Yongchareon, S.; Komosny, D. Visible light communication: A system perspective-Overview and challenges. Sensors 2019, 19, 1153. [CrossRef] [PubMed]

2. Katz, M.; Ahmed, I.; Laru, J.; Häkkinen, J. Interactive Visible Light Communications (iVLC): Supporting Wireless Communications and Smart Visual Interaction with a Common Lighting Infrastructure. In Proceedings of the 2020 South American Colloquium on Visible Light Communications (SACVC), Santiago, Chile, 4-5 June 2020; pp. 1-6.

3. Appleby, R.; Overbeeke, K. Tangible Light Interaction. In Proceedings of the Ambient Intelligence Perspectives: Selected Papers from the First International Ambient Intelligence Forum, Hradec Králové, Czech Republic, 15-16 October 2008.

4. Hartson, R.; Pyla, P.S. The UX Book: Agile UX Design for a Quality User Experience; Morgan Kaufmann: Amsterdam, The Netherlands, 2018.

5. Hornecker, E. The role of physicality in tangible and embodied interactions. Interactions 2011, 18, 19-23. [CrossRef]

6. Lee, K.R.; Goh, G.i.; Park, Y.W. Quietto: An interactive timepiece molded in concrete and milled wood. In Proceedings of the 2017 CHI Conference on Human Factors in Computing Systems, Denver, CO, USA, 6-11 May 2017; pp. 2988-2992.

7. Arif, A.S.; East, B.; DeLong, S.; Manshaei, R.; Gupta, A.; Lalwani, M.; Mazalek, A. Extending the Design Space of Tangible Objects via Low-Resolution Edge Displays. In Proceedings of the Eleventh International Conference on Tangible, Embedded, and Embodied Interaction, Yokohama, Japan, 20-23 March 2017; pp. 481-488.

8. Visser, T.; Vastenburg, M.H.; Keyson, D.V. Designing to support social connectedness: The case of SnowGlobe. Int. J. Des. 2011, 5, 129-142. 
9. Häkkilä, J.; Li, H.; Koskinen, S.; Colley, A. Connected Candles as Peripheral Emotional User Interface. In Proceedings of the 17th International Conference on Mobile and Ubiquitous Multimedia, Cairo, Egypt, 25-28 November 2018; pp. 327-333.

10. Kikin-Gil, E. The Light-Wall: Tangible user interfaces for learning systems thinking. Pers. Ubiquitous Comput. 2006, 10, 181-182. [CrossRef]

11. Sridhar, P.K.; Nanayakkara, S.; Huber, J. Towards understanding of play with augmented toys. In Proceedings of the 8th Augmented Human International Conference, Mountain View, CA, USA, 16-18 March 2017; pp. 1-4.

12. Ryokai, K.; Su, P.; Kim, E.; Rollins, B. Energybugs: Energy harvesting wearables for children. In Proceedings of the SIGCHI Conference on Human Factors in Computing Systems, Toronto, ON, Canada, 26 April-1 May 2014; pp. 1039-1048.

13. Verish, C.; Strawhacker, A.; Bers, M.; Shaer, O. CRISPEE: A Tangible Gene Editing Platform for Early Childhood. In Proceedings of the Twelfth International Conference on Tangible, Embedded, and Embodied Interaction, Stockholm, Sweden, 18-21 March 2018; pp. 101-107.

14. Gallacher, S.; O'Connor, J.; Bird, J.; Rogers, Y.; Capra, L.; Harrison, D.; Marshall, P. Mood squeezer: Lightening up the workplace through playful and lightweight interactions. In Proceedings of the 18th ACM Conference on Computer Supported Cooperative Work \& Social Computing, Vancouver, BC, Canada, 14-18 March 2015; pp. 891-902.

15. Dourish, P. Where the Action Is: The Foundations of Embodied Interaction; MIT Press: Cambridge, MA, USA, 2004.

16. Rogers, Y. Interaction design gone wild: Striving for wild theory. Interactions 2011, 18, 58-62. [CrossRef]

17. Klemmer, S.R.; Hartmann, B.; Takayama, L. How bodies matter: Five themes for interaction design. In Proceedings of the 6th Conference on Designing Interactive Systems, University Park, PA, USA, 26-28 June 2006; pp. 140-149.

18. Hornecker, E.; Buur, J. Getting a grip on tangible interaction: A framework on physical space and social interaction. In Proceedings of the SIGCHI Conference on Human Factors in Computing Systems, Montréal, QC, Canada, 22-27 April 2006; pp. 437-446.

19. Hurtienne, J. Cognition in HCI: An ongoing story. Hum. Technol. Interdiscip. J. Hum. ICT Environ. 2009, 5, 12-28.. [CrossRef]

20. Katz, M.; Järvelä, S.; Häkkinen, J. Interactive Visible Light Communications: Using Human-Light Interaction in Learning Contexts. In Proceedings of the 11th International Conference on Computer Supported Collaborative Learning, Gothenburg, Sweden, 7-11 June 2015; pp. 1-2.

21. Norman, D.A.; Draper, S.W. User Centered System Design: New Perspectives on Human-Computer Interaction; CRC Press: Mahwah, NJ, USA, 1986.

22. Bederson, B.B.; Bederson, B.B.; Shneiderman, B. The Craft of Information Visualization: Readings and Reflections; Morgan Kaufmann: San Francisco, CA, USA, 2003.

23. Rogers, Y. New theoretical approaches for human-computer interaction. Annu. Rev. Inf. Sci. Technol. 2004, 38, 87-143. [CrossRef]

24. Halverson, C.A. Activity theory and distributed cognition: Or what does CSCW need to DO with theories? Comput. Support. Coop. Work. 2002, 11, 243-267. [CrossRef]

25. Sorathia, K.; Servidio, R. Learning and experience: Teaching tangible interaction \& edutainment. Procedia Soc. Behav. Sci. 2012, 64, 265-274.

26. Verhaegh, J.; Fontijn, W.; Jacobs, A. On the benefits of tangible interfaces for educational games. In Proceedings of the 2008 Second IEEE International Conference on Digital Game and Intelligent Toy Enhanced Learning, Banff, BC, Canada, 17-19 November 2008; pp. 141-145.

27. Hemmert, F.; Hamann, S.; Wettach, R. The digital hourglass. In Proceedings of the 3rd International Conference on Tangible and Embedded Interaction, Cambridge, UK, 16-18 February 2009; pp. 19-20.

28. Matviienko, A.; Horwege, S.; Frick, L.; Ressel, C.; Boll, S. CubeLendar: Design of a Tangible Interactive Event Awareness Cube. In Proceedings of the 2016 CHI Conference Extended Abstracts on Human Factors in Computing Systems, San Jose, CA, USA, 7-12 May 2016; pp. 2601-2608.

29. Rodrigues, A.; Lopes, L.; Costa, C.; Cabral, D. LightStress: Targeting stress reduction through affective objects. In Proceedings of the Adjunct Proceedings of the 2019 ACM International Joint Conference on Pervasive and Ubiquitous Computing and Proceedings of the 2019 ACM International Symposium on Wearable Computers, London, UK, 9-13 September 2019; pp. 191-193. 
30. Gaissmaier, M.; Karlsson, A.; Aschan Eriksson, S.; Kosmack Vaara, E.; Komazec, K.; Ferneaus, Y. Designing for Workplace Safety: Exploring Interactive Textiles as Personal Alert Systems. In Proceedings of the Fourteenth International Conference on Tangible, Embedded, and Embodied Interaction, Sydney, Australia, 9-12 February 2020; pp. 53-65.

31. Saviot, L.; Brudy, F.; Houben, S. WRISTBAND. IO: Expanding input and output spaces of a Smartwatch. In Proceedings of the 2017 CHI Conference Extended Abstracts on Human Factors in Computing Systems, Denver, CO, USA, 6-11 May 2017; pp. 2025-2033.

32. Huber, J.; Steimle, J.; Liao, C.; Liu, Q.; Mühlhäuser, M. Lightbeam: Nomadic pico projector interaction with real world objects. In Proceedings of the CHI'12 Extended Abstracts on Human Factors in Computing Systems, Austin, TX, USA, 5-10 May 2012; pp. 2513-2518.

33. Jeong, K.A.; Kim, E.; Kim, T.; Suk, H.J. c. light: A Tool for Exploring Light Properties in Early Design Stage. In Proceedings of the 2018 CHI Conference on Human Factors in Computing Systems, Montreal, QC, Canada, 21-26 April 2018; pp. 1-12.

34. Miller, H.C.; Kubicki, S.; Kolski, C.; Naveteur, J. Implementation of the stroop task using an interactive table: An experimental study. In Proceedings of the CHI'13 Extended Abstracts on Human Factors in Computing Systems, Paris, France, 27 April-2 May 2013; pp. 253-258.

35. Pla, P.; Maes, P. Display blocks: Cubic displays for multi-perspective visualization. In Proceedings of the CHI'12 Extended Abstracts on Human Factors in Computing Systems, Austin, TX, USA, 5-10 May 2012; pp. 2015-2020.

36. Fujii, J.; Matsunobu, T.; Kakehi, Y. COLORISE: Shape-and Color-Changing Pixels with Inflatable Elastomers and Interactions. In Proceedings of the Twelfth International Conference on Tangible, Embedded, and Embodied Interaction, Stockholm, Sweden, 18-21 March 2018; pp. 199-204.

37. Kim, Y. Weight of Data. In Extended Abstracts of the 2018 CHI Conference on Human Factors in Computing Systems; Association for Computing Machinery: New York, NY, USA, 2018; pp. 1-6. [CrossRef]

38. Schilling, M.L.; Wakkary, R.; Odom, W. Focus framework: Tracking prototypes' back-talk. In Proceedings of the Twelfth International Conference on Tangible, Embedded, and Embodied Interaction, Stockholm, Sweden, 18-21 March 2018; pp. 684-693.

39. Vandenberghe, B.; Gerling, K.; Geurts, L.; Vanden Abeele, V. Skweezee for Processing: A Software Library to Make Squeeze Interactions. In Proceedings of the Thirteenth International Conference on Tangible, Embedded, and Embodied Interaction, Tempe, AZ, USA, 17-20 March 2019; pp. 375-381.

40. Flemings, M.; Kazmi, S.; Pak, R.; Shaer, O. Crimson wave: Shedding light on menstrual health. In Proceedings of the Twelfth International Conference on Tangible, Embedded, and Embodied Interaction, Stockholm, Sweden, 18-21 March 2018; pp. 343-348.

41. Makino, Y.; Kakehi, Y. Metamorphic light: A tabletop tangible interface using deformation of plain paper. In Proceedings of the ACM SIGGRAPH 2011 Posters, Vancouver, BC, Canada, 7-11 August 2011; p. 1.

42. Harrison, C.; Ramamurthy, S.; Hudson, S.E. On-body interaction: Armed and dangerous. In Proceedings of the Sixth International Conference on Tangible, Embedded and Embodied Interaction, Kingston, ON, Canada, 19-22 February 2012; pp. 69-76.

43. Kao, H.L.; Chuah, M.Y.; Degen, M.; Tucker, J.; Ishii, H. LightBundle: Grasping light through plant-inspired interactions. In Proceedings of the CHI'14 Extended Abstracts on Human Factors in Computing Systems, Toronto, ON, Canada, 26 April-1 May 2014; pp. 1849-1854.

44. Najafizadeh, L.; Kang, S.; Froehlich, J.E. "I Like This Shirt” Exploring the Translation of Social Mechanisms in the Virtual World into Physical Experiences. In Proceedings of the 33rd Annual ACM Conference Extended Abstracts on Human Factors in Computing Systems, Seoul, Korea, 18-23 April 2015; pp. 1929-1934.

45. Sørensen, T.; Andersen, O.D.; Merritt, T. “Tangible Lights" In-Air Gestural Control of Home Lighting. In Proceedings of the Ninth International Conference on Tangible, Embedded, and Embodied Interaction, Stanford, CA, USA, 15-19 January 2015; pp. 727-732.

46. Tong, Y.; Sikorska, J.E.; Silva, C.D.; Singh, M.; Mhatre, S. LightShare: Sharing illumination the tangible way. In Proceedings of the 2015 British HCI Conference, Lincoln, UK, 13-17 July 2015; pp. 305-306.

47. van de Werff, T.; van Lotringen, C.; van Essen, H.; Eggen, B. Design Considerations for Interactive Office Lighting: Interface Characteristics, Shared and Hybrid Control. In Proceedings of the 2019 CHI Conference on Human Factors in Computing Systems, Glasgow, UK, 4-9 May 2019; pp. 1-14. 
48. Marz, A.; Brito, C.; Vargas, R. Lumigami. In Proceedings of the 2019 14th ACM/IEEE International Conference on Human-Robot Interaction (HRI), Daegu, Korea, 11-14 March 2019; pp. 761-762.

49. Van Rooij, T.; Bakker, S. Plux: Exploring light settings through hybrid control. In Proceedings of the Twelfth International Conference on Tangible, Embedded, and Embodied Interaction, Stockholm, Sweden, 18-21 March 2018; pp. 205-210.

50. Verweij, D.; Esteves, A.; Bakker, S.; Khan, V.J. Designing motion matching for real-world applications: Lessons from realistic deployments. In Proceedings of the Thirteenth International Conference on Tangible, Embedded, and Embodied Interaction, Tempe, AZ, USA, 17-20 March 2019; pp. 645-656.

51. Alonso, M.B.; Keyson, D.V. MusicCube: A physical experience with digital music. Pers. Ubiquitous Comput. 2006, 10, 163-165. [CrossRef]

52. Ying, F.T.; Cai, J.X.; Wang, Q.; Tao, Y.; Chen, C.; Wang, Y.N.; Yao, C. Cultural Design Method on Multi-sensor Technology Design: A Case of Interactive Lamp Design. In Applied Mechanics and Materials; Trans Tech Publ.: Stafa-Zurich, Switzerland, 2013; Volume 321, pp. 553-557.

53. Leigh, S.W.; Roseway, A.; Paradiso, A. Remnance of Form: Altered Reflection of Physical Reality. In Proceedings of the Ninth International Conference on Tangible, Embedded, and Embodied Interaction, Stanford, CA, USA, 15-19 January 2015; pp. 411-412.

54. Ross, P.R.; Wensveen, S.A. Designing aesthetics of behavior in interaction: Using aesthetic experience as a mechanism for design. Int. J. Des. 2010, 4, 3-13.

55. Lappalainen, T.; Korpela, J.; Colley, A.; Häkkilä, J. Experiential tangible UI for controlling lighting. In Proceedings of the 2016 ACM International Joint Conference on Pervasive and Ubiquitous Computing: Adjunct, Heidelberg, Germany, 12-16 September 2016; pp. 317-320.

56. Shi, Y.; Ying, F.; Yu, J.; Jia, P. Tangible Light: Back to metaphor-based interaction. In Proceedings of the 2010 International Symposium on Computational Intelligence and Design, Hangzhou, China, 29-31 October 2010; Volume 1, pp. 53-55.

57. Wouters, N.; Hunt, T.; Dziemidowicz, O.; Hiscock, R.; Vetere, F. Media Architecture in Knowledge and Innovation Districts: Designing a Canvas for Research, Culture and Collaboration. In Proceedings of the 4th Media Architecture Biennale Conference, Beijing, China, 13-16 November 2018; pp. 35-44.

58. Cao, Y.Y.; Okude, N. Scented pebbles: Interactive ambient experience with smell and lighting. In Proceedings of the Ninth International Conference on Tangible, Embedded, and Embodied Interaction, Stanford, CA, USA, 15-19 January 2015; pp. 409-410.

59. Chew, L.; Cochrane, K.A.; Hespanhol, L.; Loke, L. Sounds of Infinity: Playful Illusions with Performative Interaction. In Proceedings of the Fourteenth International Conference on Tangible, Embedded, and Embodied Interaction, Sydney, Australia, 9-12 February 2020; pp. 669-675.

60. Bakker, S.; van den Hoven, E.; Eggen, B. FireFlies: Physical peripheral interaction design for the everyday routine of primary school teachers. In Proceedings of the 7th International Conference on Tangible, Embedded and Embodied Interaction, Barcelona, Spain, 10-13 February 2013; pp. 57-64.

61. Alavi, H.S.; Dillenbourg, P.; Kaplan, F. Distributed awareness for class orchestration. In Proceedings of the European Conference on Technology Enhanced Learning, Nice, France, 29 September-2 October 2009; pp. 211-225.

62. Alavi, H.S.; Dillenbourg, P. An ambient awareness tool for supporting supervised collaborative problem solving. IEEE Trans. Learn. Technol. 2012, 5, 264-274. [CrossRef]

63. Bekker, T.; Sturm, J. Stimulating physical and social activity through open-ended play. In Proceedings of the 8th International Conference on Interaction Design and Children, Como, Italy, 3-5 June 2009; pp. 309-312.

64. Buechley, L.; Eisenberg, M. Boda blocks: A collaborative tool for exploring tangible three-dimensional cellular automata. In Proceedings of the 8th Iternational Conference on Computer Supported Collaborative Learning, New Brunswick, NJ, USA, 16-21 July 2007; pp. 102-104.

65. Dijkhuis, S.; Offermans, S.; Bakker, S. Vuur: Exploring shared interaction with light. Machines 2015, 3, $296-316$. [CrossRef]

66. Maquil, V.; Moll, C.; Schwartz, L.; Hermen, J. Kniwwelino: A Lightweight and WiFi Enabled Prototyping Platform for Children. In Proceedings of the Twelfth International Conference on Tangible, Embedded, and Embodied Interaction, Stockholm, Sweden, 18-21 March 2018; pp. 94-100. 
67. Salihodžic, H.; Zilberburg, K.; Chakhmaghi, N.; Güldenpfennig, F.; Fikar, P.; Ganhör, R. LightSight: A Dice to Meet the Eyes. In Proceedings of the 2018 ACM Conference Companion Publication on Designing Interactive Systems, Hong Kong, China, 9-13 June 2018; pp. 331-334.

68. Vazquez, V.; Cibrian, F.L.; Tentori, M. StretchyStars: A multitouch elastic display to support cooperative play among preschoolers. Pers. Ubiquitous Comput. 2019, 23, 99-115. [CrossRef]

69. Müller, H.; Pieper, C.; Heuten, W.; Boll, S. It's Not That Long! Helping Children to Understand Time with an Ambient Light Display. In Proceedings of the The 15th International Conference on Interaction Design and Children, Manchester, UK, 21-24 June 2016; pp. 356-366.

70. Hallam, J.; Zheng, C.; Posner, N.; Ericson, H.; Swarts, M.; Do, E.Y.L. The Light Orchard: An immersive display platform for collaborative tangible interaction. In Proceedings of the 2017 ACM International Joint Conference on Pervasive and Ubiquitous Computing and Proceedings of the 2017 ACM International Symposium on Wearable Computers, Maui, HI, USA, 13-15 September 2017; pp. 245-248.

71. Zheng, C.; Hallam, J.; Posner, N.; Ericson, H.; Swarts, M.; Do, E.Y.L. Game of Light: Modeling Diversity Through Participatory Interaction. In Proceedings of the $2017 \mathrm{CHI}$ Conference Extended Abstracts on Human Factors in Computing Systems, Denver, CO, USA, 6-11 May 2017; pp. 417-420.

72. Hoggenmueller, M.; Hespanhol, L. Woodie. An Urban Robot for Embodied Hybrid Placemaking. Proceedings of the Fourteenth International Conference on Tangible, Embedded, and Embodied Interaction, Sydney, Australia, 9-12 February 2020; pp. 617-624.

73. Sugiura, Y.; Kakehi, G.; Withana, A.; Lee, C.; Sakamoto, D.; Sugimoto, M.; Inami, M.; Igarashi, T. Detecting shape deformation of soft objects using directional photoreflectivity measurement. In Proceedings of the 24th Annual ACM Symposium on User Interface Software and Technology, Santa Barbara, CA, USA, 16-19 October 2011; pp. 509-516.

74. Garcia, T.; Brown, S.; Dev, S. TUIst: A collaborative and computationally enhanced game board. In Proceedings of the Twelfth International Conference on Tangible, Embedded, and Embodied Interaction, Stockholm, Sweden, 18-21 March 2018; pp. 337-342.

75. Chhikara, A.; Hespanhol, L. Rayuela: Delivering Serious Information Through Playful Interactive Installations. In Proceedings of the Fourteenth International Conference on Tangible, Embedded, and Embodied Interaction, Sydney, Australia, 9-12 February 2020; pp. 661-667.

76. Downey, C.; Kamel, S.W. Storytime with Hue: An innovative approach to storytelling where storytellers control a dynamic lighting environment. In Proceedings of the TEI'16: Tenth International Conference on Tangible, Embedded, and Embodied Interaction, Eindhoven, The Netherlands, 14-17 February 2016; pp. 422-427.

77. Wang, P.; Koh, R.K.; Boucharenc, C.G.; Yen, C.C. Lights Out: An Interactive Tangible Game for Training of Post-Stroke Reaching. In Proceedings of the 2016 CHI Conference Extended Abstracts on Human Factors in Computing Systems, San Jose, CA, USA, 7-12 May 2016; pp. 1937-1944.

78. Yang, Z.; Jie, S.; Shiqi, L.; Ping, C.; Shengjia, N. Tangible Interactive Upper Limb Training Device. In Proceedings of the 2018 ACM Conference Companion Publication on Designing Interactive Systems, Hong Kong, China, 9-13 June 2018; pp. 1-5.

79. Yang, Z.; Jie, S.; Yanhao, J.; Yixuan, B. Interactive Tabletop Arm Reaching Exercise. In Proceedings of the Thirteenth International Conference on Tangible, Embedded, and Embodied Interaction, Tempe, AZ, USA, 17-20 March 2019; pp. 423-428.

80. Eun, J.; Lee, Y.; Lee, J.; Lee, J.; Lee, W.S.; Lee, Z. HabitStar: An Interactive Ambient Lighting to Help Users Improve Habits. In Proceedings of the 33rd Annual ACM Conference Extended Abstracts on Human Factors in Computing Systems, Seoul, Korea, 18-23 April 2015; pp. 1489-1494.

81. Lee, J.; Lee, J.Y.; Kim, S.W.; Cho, J.D. D-TOX: Inducing Digital Detox for Nighttime via Smart Lamp Applied Gamification. In Proceedings of the 2017 Conference on Interaction Design and Children, Stanford, CA, USA, 27-30 June 2017; pp. 497-502.

82. Chiarello, F. Playing With Light: A Game Based Approach to Learn Photonics in FabLabs. In Proceedings of the ECGBL 2018 12th European Conference on Game-Based Learning, Sophia Antipolis, France, 4-5 October 2018; p. 31.

83. Ogawa, H.; Ando, N.; Onodera, S. SmallConnection: Designing of tangible communication media over networks. In Proceedings of the 13th Annual ACM International Conference on Multimedia, Singapore, 6-11 November 2005; pp. 1073-1074. 
84. Schmeer, J.; Baffi, T. Touch trace mirror: Asynchronous, collaborative messaging as a concept for creating a relatedness experience. In Proceedings of the Fifth International Conference on Tangible, Embedded, and Embodied Interaction, Cambridge, MA, USA, 24-27 January 2010; pp. 303-304.

85. Deng, H.; Li, J.; Sayegh, A.; Birolini, S.; Andreani, S. Twinkle: A flying lighting companion for urban safety. In Proceedings of the Twelfth International Conference on Tangible, Embedded, and Embodied Interaction, Stockholm, Sweden, 18-21 March 2018; pp. 567-573.

86. Paredes, P.; Ko, R.; Calle-Ortiz, E.; Canny, J.; Hartmann, B.; Niemeyer, G. Fiat-lux: Interactive urban lights for combining positive emotion and efficiency. In Proceedings of the 2016 ACM Conference on Designing Interactive Systems, Brisbane, Australia, 4-8 June 2016; pp. 785-795.

87. Angelini, L.; Caon, M.; Lalanne, D.; Abou khaled, O.; Mugellini, E. Towards an anthropomorphic lamp for affective interaction. In Proceedings of the Ninth International Conference on Tangible, Embedded, and Embodied Interaction, Stanford, CA, USA, 15-19 January 2015; pp. 661-666.

88. Shen, X.; George, M.; Hernandez, S.; Park, A.; Liu, Y.; Ishii, H. Mole Messenger: Pushable Interfaces for Connecting Family at a Distance. In Proceedings of the Thirteenth International Conference on Tangible, Embedded, and Embodied Interaction, Tempe, AZ, USA, 17-20 March 2019; pp. 269-274.

89. Turan, S.G.; Ozaltin, S.R.; Yantac, A.E. Designing calmworklight: Signaling Focus with a Desk Light. In Proceedings of the Companion Publication of the 2019 on Designing Interactive Systems Conference 2019 Companion, San Diego, CA, USA, 23-28 June 2019; pp. 301-305.

90. Pörtner, A.; Schröder, L.; Rasch, R.; Sprute, D.; Hoffmann, M.; König, M. The Power of Color: A Study on the Effective Use of Colored Light in Human-Robot Interaction. In Proceedings of the 2018 IEEE/RSJ International Conference on Intelligent Robots and Systems (IROS), Madrid, Spain, 1-5 October 2018; pp. 3395-3402.

91. Freeman, E.; Brewster, S.; Lantz, V. Interactive light feedback: Illuminating Above-Device gesture interfaces. In Proceedings of the IFIP Conference on Human-Computer Interaction, Bamberg, Germany, 14-18 September 2015; pp. 478-481.

92. Huang, Y.C.A.; Tsai, C.C.; Chang, T.W.; Tsai, P.Y.; Hung, T.H.; Chen, J.J. Blowing light: Green-based interaction design. In Proceedings of the International Conference on Human-Computer Interaction, Orlando, FL, USA, 9-14 July 2011; pp. 82-91.

93. Mao, C.C.; Liu, K.H.; Chiu, W.C.; Lin, C.L.; Chen, C.H. Citycell: An interactive OLED lighting system in public space. In Proceedings of the 3rd International Conference on Communication and Information Processing, Tokyo, Japan, 24-26 November 2017; pp. 490-494.

94. Chang, A.; Resner, B.; Koerner, B.; Wang, X.; Ishii, H. LumiTouch: An emotional communication device. In Proceedings of the CHI'01 Extended Abstracts on Human Factors in Computing Systems, Seattle, WA, USA, 31 March-5 April 2001; pp. 313-314.

95. Offermans, S.; Van Essen, H.; Eggen, J. User interaction with everyday lighting systems. Pers. Ubiquitous Comput. 2014, 18, 2035-2055. [CrossRef]

96. Ueki, A.; Watanabe, K.; Inakage, M. CREATUREs-designing of interactive interior lamps. In Proceedings of the ACM SIGGRAPH 2006 Sketches, Boston, MA, USA, 30 July-3 August 2006; p. 36-es.

97. Lehrer, N.; Tinapple, D.; Koziupa, T.; Chen, M.; Kidane, A.; Rajko, S.; Wallis, I.; Baran, M.; Lorig, D.; Siwiak, D.; et al. Thrii. In Proceedings of the 18th ACM International Conference on Multimedia, Firenze, Italy, 25-29 October 2010; pp. 1425-1428.

98. Rossmy, B.; Wiethoff, A. COMB-Shape as a Meaningful Element of Interaction. In Proceedings of the Thirteenth International Conference on Tangible, Embedded, and Embodied Interaction, Tempe, AZ, USA, 17-20 March 2019; pp. 287-295.

99. Schiettecatte, B.; Vanderdonckt, J. AudioCubes: A distributed cube tangible interface based on interaction range for sound design. In Proceedings of the 2nd International Conference on Tangible and Embedded Interaction, Bonn, Germany, 18-20 February 2008; pp. 3-10.

100. Qin, Q.; Rohs, M.; Kratz, S. Dynamic ambient lighting for mobile devices. In Proceedings of the 24th annual ACM Symposium Adjunct on User Interface Software and Technology, Santa Barbara, CA, USA, 16-19 October 2011; pp. 51-52.

101. Kwon, H.; Kim, H.; Lee, W. Intangibles wear materiality via material composition. Pers. Ubiquitous Comput. 2014, 18, 651-669. [CrossRef] 
102. Czuszynski, K.; Ruminski, J. Towards Contactless, Hand Gestures-Based Control of Devices. In Proceedings of the IECON 2018 - 44th Annual Conference of the IEEE Industrial Electronics Society, Washington, DC, USA, 21-23 October 2018; pp. 3298-3303.

103. Choi, Y.; Kim, J.; Pan, P.; Jeung, J. The considerable elements of the emotion expression using lights in apparel types. In Proceedings of the 4th International Conference on Mobile Technology, Applications, and Systems and the 1st International Symposium on Computer Human Interaction in Mobile Technology, Singapore, 10-12 September 2007; pp. 662-666.

104. Alex, D.; Gorur, P.; Amrutur, B.; Ramakrishnan, K. LampTop: Touch detection for a projector-camera system based on shape classification. In Proceedings of the 2013 ACM International Conference on Interactive Tabletops and Surfaces, St. Andrews, UK, 6-9 October 2013; pp. 429-432.

105. Matrosov, M.; Volkova, O.; Tsetserukou, D. LightAir: A novel system for tangible communication with quadcopters using foot gestures and projected image. In Proceedings of the ACM SIGGRAPH 2016 Emerging Technologies, Anaheim, CA, USA, 24-28 July 2016; pp. 1-2.

106. Fortmann, J.; Root, E.; Boll, S.; Heuten, W. Tangible apps bracelet: Designing modular wrist-worn digital jewellery for multiple purposes. In Proceedings of the 2016 ACM Conference on Designing Interactive Systems, Brisbane, Australia, 4-8 June 2016; pp. 841-852.

107. Oki, M.; Tsukada, K. Sparklry: Designing "Sparkle" of Interactive Jewelry. In Proceedings of the Eleventh International Conference on Tangible, Embedded, and Embodied Interaction, Yokohama, Japan, 20-23 March 2017; pp. 647-651.

108. Fastnacht, T.; Aispuro, A.O.; Marschall, J.; Fischer, P.T.; Zierold, S.; Hornecker, E. Sonnengarten: Urban light installation with human-plant interaction. In Proceedings of the 2016 ACM International Joint Conference on Pervasive and Ubiquitous Computing: Adjunct, Heidelberg, Germany, 12-16 September 2016; pp. 53-56.

109. Cuculo, V.; Lanzarotti, R.; Boccignone, G. The color of smiling: Computational synaesthesia of facial expressions. In Proceedings of the International Conference on Image Analysis and Processing, Cagliari, Italy, 6-8 September 2015; pp. 203-214.

110. Seaborn, K.; Antle, A.N. The tiresias effect: Feedforward using light versus temperature in a tangible user interface. In Proceedings of the CHI'10 Extended Abstracts on Human Factors in Computing Systems, Atlanta, GA, USA, 10-15 April 2010; pp. 3427-3432.

111. Liu, Y.; Lee, Y.H.; Chuang, Y.; Liang, R.H.; Chen, L.L. Designing the expressive point lights to enhance user's situated awareness of smart systems. In Proceedings of the 2017 ACM Conference Companion Publication on Designing Interactive Systems, Edinburgh, UK, 10-14 June 2017; pp. 333-336.

112. Yamaoka, J.; Kakehi, Y. NeonDough: Crafting with interactive lighted clay. In Proceedings of the ACM SIGGRAPH 2012, Los Angeles, CA, USA, 5-9 August 2012; Association for Computing Machinery: New York, NY, USA, 2012; p. 1.

113. Yamamoto, Y.; Uchiyama, H.; Kakehi, Y. onNote: Playing printed music scores as a musical instrument. In Proceedings of the 24th Annual ACM Symposium on User Interface Software and Technology, Santa Barbara, CA, USA, 16-19 October 2011; pp. 413-422.

114. Lenz, E.; Diefenbach, S.; Hassenzahl, M. Aesthetics of Interaction: A Literature Synthesis. In Proceedings of the 8th Nordic Conference on Human-Computer Interaction: Fun, Fast, Foundational (NordiCHI '14); Association for Computing Machinery: New York, NY, USA, 2014; pp. 628-637, doi:10.1145/2639189.2639198. [CrossRef]

115. Nofal, E.; Panagiotidou, G.; Reffat, R.M.; Hameeuw, H.; Boschloos, V.; Moere, A.V. Situated Tangible Gamification of Heritage for Supporting Collaborative Learning of Young Museum Visitors. J. Comput. Cult. Herit. 2020, 13, doi:10.1145/3350427. [CrossRef]

116. Nofal, E.; Reffat, R.M.; Boschloos, V.; Hameeuw, H.; Moere, A.V. The role of tangible interaction to communicate tacit knowledge of built heritage. Heritage 2018, 1, 414-436. [CrossRef]

117. Brave, S.; Ishii, H.; Dahley, A. Tangible interfaces for remote collaboration and communication. In Proceedings of the 1998 ACM Conference on Computer Supported Cooperative Work, Seattle, WA, USA, 14-18 November 1998; pp. 169-178.

118. Underkoffler, J.; Ishii, H. Illuminating light: An optical design tool with a luminous-tangible interface. In Proceedings of the SIGCHI Conference on Human Factors in Computing Systems, Los Angeles, CA, USA, 18-23 April 1998; pp. 542-549. 
119. Brave, S.; Dahley, A. inTouch: A medium for haptic interpersonal communication. In Proceedings of the CHI'97 Extended Abstracts on Human Factors in Computing Systems, Atlanta, GA, USA, $22-27$ March 1997; pp. 363-364.

120. Leithinger, D.; Follmer, S.; Olwal, A.; Ishii, H. Physical telepresence: Shape capture and display for embodied, computer-mediated remote collaboration. In Proceedings of the 27th Annual ACM Symposium on User Interface Software and Technology, Honolulu, HI, USA, 5-8 October 2014; pp. 461-470.

121. Tsui, K.M.; Desai, M.; Yanco, H.A.; Uhlik, C. Exploring use cases for telepresence robots. In Proceedings of the 2011 6th ACM/IEEE International Conference on Human-Robot Interaction (HRI), Lausanne, Switzerland, 8-11 March 2011; pp. 11-18.

122. Narumi, T.; Hiyama, A.; Tanikawa, T.; Hirose, M. Inter-glow: Interaction by controlling light. In Proceedings of the international Conference on Advances in Computer Entertainment Technology, Salzburg, Austria, 13-15 June 2007; pp. 276-277.

(C) 2020 by the authors. Licensee MDPI, Basel, Switzerland. This article is an open access article distributed under the terms and conditions of the Creative Commons Attribution (CC BY) license (http://creativecommons.org/licenses/by/4.0/). 